\title{
Suppressing and initiation of multipactor discharge on a dielectric by an external dc bias
}

\author{
O. A. Ivanov, M. A. Lobaev, V. A. Isaev, and A. L. Vikharev \\ Institute of Applied Physics RAS, 603950 Nizhny Novgorod, Russia
}

(Received 3 June 2009; published 25 February 2010)

\begin{abstract}
The results of studying experimentally the influence of an external dc electric field on the multipactor threshold on the surface of a dielectric (quartz) are presented. In the experiments, a high- $Q$-factor microwave resonator was excited at the $\mathrm{TE}_{012}$ mode in the 3 - $\mathrm{cm}$ wavelength band. The dependence of the breakdown threshold on the value and direction of the electrostatic field is determined. It is found that the external dc field repulsing the electrons from the dielectric surface increases the threshold significantly, whereas the field attracting the electrons decreases it. It is shown that one can manage the multipactor efficiently, namely suppress or initiate it, by changing the direction and intensity of the dc field.
\end{abstract}

PACS numbers: 52.80.Pi, 52.80.Vp

The term "multipactor discharge" means a specific form of the discharge occurring in vacuum near a dielectric or metal surface under the effect of a high-frequency electromagnetic field [1]. The mechanism of the discharge evolution is associated with the avalanche-type multiplication of free electrons as they are bombarding the surface. Currently, the multipactor is regarded as an extremely undesirable phenomenon, which prevents generation and release of high-power electromagnetic radiation in microwave generators and impedes efficient operation of space communication systems. For example, a multipactor discharge on the surface of a dielectric window in a highpower microwave vacuum device may destroy the window. Therefore, studying the conditions of multipactor initiation and suppression seems to be rather important, specifically, for the development of output windows used in high-power microwave generators, accelerating dielectric structures of electron-positron accelerators, and active compressors of microwave pulses [2-4].

Preconditions of multipactor initiation are the presence of an external field, which brings the electrons back to the surface, and the excess of the electron's energy over the value, at which the secondary electron-emission yield is equal to unity. Therefore, all existing methods of multipactor suppression can be subdivided into two categories. The first category includes the methods allowing one to decrease the energy and secondary emission of electrons. Such methods include changing the geometry of the waveguide system (electromagnetic field structure) in order to reduce high-frequency fields on the dielectric surface [4,5] or modifying the dielectric surface by using special corrugations [6] or depositing films with low secondary electron emission yields on it [7-9]. However, in some cases it is not possible to change the system geometry, and the method of surface modification is complicated and not always produces the desired results. The other group comprises the methods, which implies production of static electric $[10,11]$ or magnetic $[9,12]$ fields near the dielectric surface, which prevent the electrons getting back to it. Indeed, by generating an external electron-repulsing field near the surface, one can stop the initiation of an electron avalanche regardless of the value of the secondary electron-emission ratio of the surface material. The possibility to use an electrostatic field to suppress the multipactor on the surface of a window in coaxial devices inputting microwaves into accelerating structures was considered in papers $[11,13]$. The external electric field was produced by applying a voltage between the central and external electrodes of the coaxial line. In the considered case, both fields, highfrequency and electrostatic ones, were directed normally to the dielectric surface. Multipactor suppression was observed only in a narrow range of parameters, which is determined by the ratio of the microwave power and the voltage.

The most common theoretical models of the multipactor $[14,15]$ employ the approximation of a homogenous rf electric field, which is parallel to the dielectric surface, and assume that there is an external static field (electric or magnetic) driving the electrons back to the surface. Figure 1 shows the schematic of the multipactor discharge on dielectric in parallel rf and normal dc fields. The energy of the electrons bombarding the surface is determined by the intensity of the microwave field and the time of their getting back to the surface under the effect of static fields $[14,15]$. Calculations show that the breakdown threshold is strongly dependent on the value of the initial electrostatic field driving the electrons back. At the same time, as far as

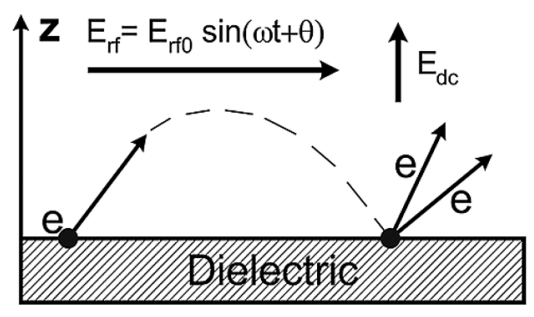

FIG. 1. Schematic of a multipactor on dielectric in parallel $\mathrm{rf}$ and normal dc fields. 
we know, the influence of the external electrostatic field on the multipactor threshold has not yet been studied purposefully in experiments.

Our paper presents the results of studying the influence of an external dc bias on the multipactor threshold on the dielectric surface. The experiments were performed "classically," i.e., the electric field of the microwaves is parallel to the dielectric surface, and the external electrostatic field is directed normally to it.

The scheme of the experimental setup is shown in Fig. 2. The setup was based on a resonator cavity formed by a cylindrical waveguide $45 \mathrm{~mm}$ in diameter and excited at a frequency of $9.4 \mathrm{GHz}$ (the $\mathrm{TE}_{012}$ mode), an iris diaphragm (7), and a plane T-shaped copper electrode (11). The electrodes had no Ohmic contact with the resonator walls, but its diameter was calculated so as to ensure a close-tounity reflection coefficient for the $\mathrm{TE}_{01}$ mode. The distance between the diaphragm and the electrode (the length of the resonator) could be changed from the outside by means of a special movable piston (14). The electrode was fed with a potential from a low-current high-voltage source (18 in Fig. 1), which allowed one to regulate the voltage in the range from $U=0$ until $U= \pm 20 \mathrm{kV}$. Quartz disks with different thicknesses were installed in an antinode of standing wave in the resonator. The measured loaded $Q$ factor of the resonator depending on the thickness of the studied disk amounted to $Q_{L}=(0.9-1.3) \times 10^{4}$. Such a $Q$ factor ensured high intensities of the electric field at the dielectric surface even for a low level of the input rf power. The resonator was installed in a vacuum chamber pumped off to the pressure $p \approx 10^{-6}$ Torr. The rf pulses were sent to the resonator input via a mode converter, which formed the $\mathrm{TE}_{01}$ mode of the circular waveguide and a microwave window (6). Microwaves were produced by a magnetron

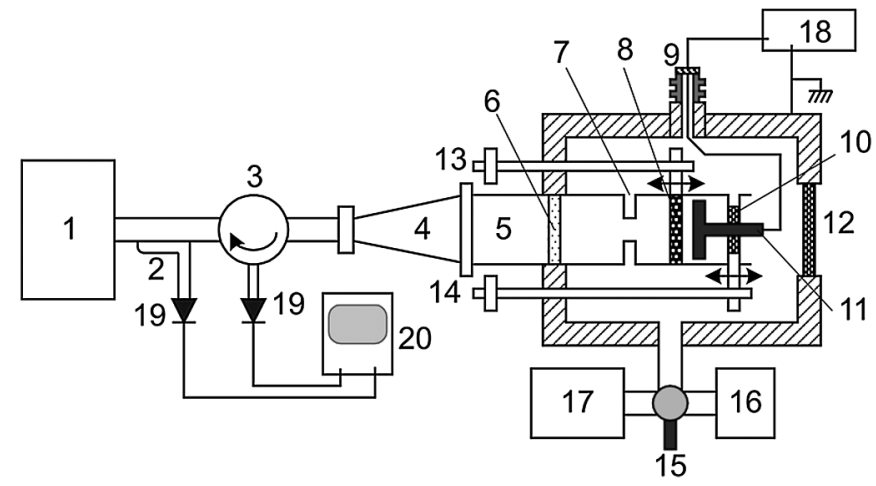

FIG. 2. Scheme of the experimental setup: 1-magnetron, 2directional coupler, 3-circulator, 4-mode converter, 5-circular waveguide, 6-microwave window, 7-diaphragm, 8studied dielectric disk, 9-high-voltage input, 10-insulator, 11-electrode (back wall of the resonator), 12-observation window, 13 and 14-disk and electrode transfer mechanisms, respectively, 15-pressure gauge, 16-mechanical pump, 17ion pump, 18-high-voltage source, 19-microwave detector, and 20 -oscilloscope.
(1) generating a power of up to $250 \mathrm{~kW}$ in pulses with the duration $\tau=1-6 \mu \mathrm{s}$ and a pulse repetition rate of up to $20 \mathrm{~Hz}$. The resonator was tuned to the magnetron frequency by changing the length of the resonator.

It was shown in $[16,17]$ theoretically and experimentally that multipactor can take place on a dielectric surface in strong electromagnetic fields even in the absence of external static fields, since an inhomogeneous microwave field can by itself ensure the return of the emitted electrons to the surface. Indeed, in an inhomogeneous microwave field, an electron is affected by the averaged ponderomotive force $F_{\phi}$, which is proportional to the gradient of the $\mathrm{rf}$ electric field [18]. The intensity of the potential electric field $E_{\phi}$ near the dielectric surface, which is equivalent to the above-mentioned force, can be written as

$$
E_{\phi}=\frac{F_{\phi}}{-e}=\frac{e}{4 m \omega^{2}} \nabla\left|E_{s}\right|^{2},
$$

where $e$ and $m$ are the electron charge and mass, $\omega$ is the frequency of microwaves, and $E_{s}$ is the intensity of the microwave field on the disk surface. In the absence of an external electrostatic field, this force may either drive the emitted electrons back to the surface or repulse them depending on the gradient sign. Therefore, in the experimental conditions, the electric field $E_{n}$, which was normal to the dielectric surface, was determined by the superposition of the external dc field $E_{z}$ and the axial component of effective field $E_{\phi z} \sim \nabla_{z}\left|E_{s}\right|^{2}$ [Eq. (1)] formed by the gradient of the rf potential, $E_{n}=E_{\phi z} \pm E_{z}$.

In the experiment, we used polished quartz disks having the surface roughness $R_{a}=0.1 \mu \mathrm{m}$ and three different thicknesses, namely, $d_{1}=2.3 \mathrm{~mm}, d_{2}=4.2 \mathrm{~mm}$, and $d_{3}=6 \mathrm{~mm}$. The disk thickness was calculated to provide three different ponderomotive force configurations on the disk surface facing the electrode: (1) driving the electrons back to the surface $\left(d_{1}\right),(2)$ no force $\left(d_{2}\right),(3)$ the electrons are repulsed $\left(d_{3}\right)$. The other surface of the disk was installed at a node of the standing wave. Thus, the conditions for the multipactor initiation were created on only one side of the disk with a specified direction and value of the ponderomotive force. The characteristic structure of the electric field in the resonator and distribution of the amplitude of the microwave field along axis $z$ in the maximum of the radial distribution of the $\mathrm{TE}_{012}$ mode for disks having different thicknesses are shown in Fig. 3. The calculations were performed by the finite difference time domain method [19].

In the experiments, we studied the influence of the value and direction of the electrostatic field generated by means of the electrode on the multipactor threshold. At the initial stage, the structure of the electrostatic field lines in the resonator was calculated for each disk, Fig. 4. The shape of the electrode was chosen such as to generate a quasihomogenous electrostatic field mainly directed normally to the surface of the dielectric disk. The ratio of the transverse 


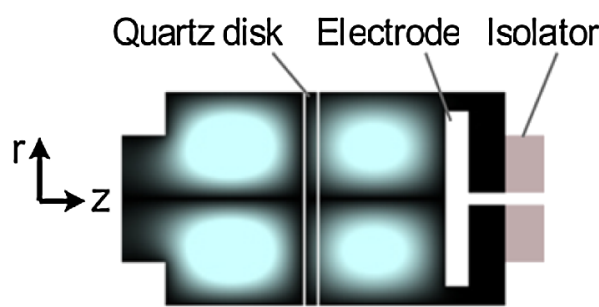

1a)

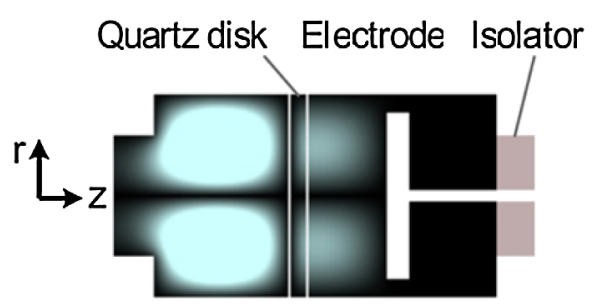

1b)

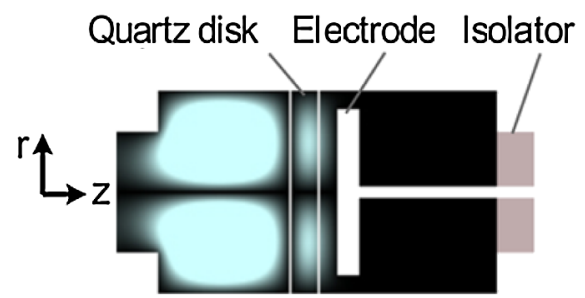

1c)
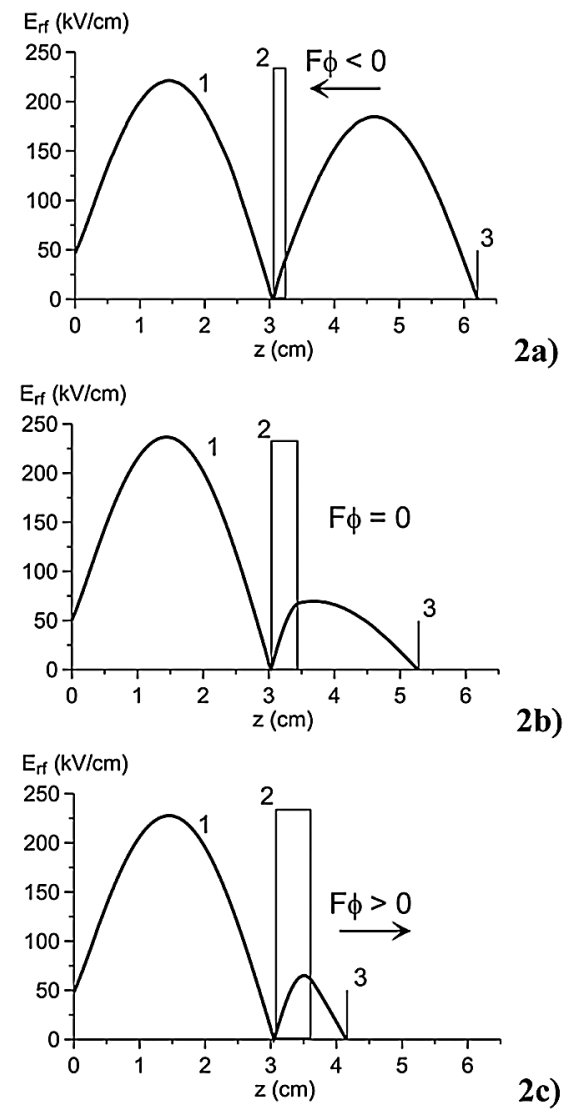

FIG. 3. (Color) Instantaneous distribution of the microwave field in the resonator for disks having different thicknesses $\left(1 \mathrm{a}-d_{1}=\right.$ $2.3 \mathrm{~mm}, 1 \mathrm{~b}-d_{2}=4.2 \mathrm{~mm}, 1 \mathrm{c}-d_{3}=6 \mathrm{~mm}$ ) and (2a-2c) dependence of the amplitude of the rf electric field at the maximum of the $\mathrm{TE}_{012}$ mode on the longitudinal coordinate for an incident power of $100 \mathrm{~kW} .1$-amplitude of the rf field, 2-quartz disk, 3- electrode (back wall of the resonator).

and the longitudinal component of the dc field on the surface of the dielectric at the maximum of the $\mathrm{TE}_{012}$ mode amounted to $E_{r} / E_{z}=0.4,0.29$, and 0.12 for the disks $d_{1}, d_{2}$, and $d_{3}$, respectively.

The distance between the disk and the electrode was assumed corresponding to the excitation of the $\mathrm{TE}_{012}$ mode in the resonator and amounted to 30,18 , or $7 \mathrm{~mm}$, respectively, depending on the disk thickness. For the thin disk, the maximum intensity of the electrostatic field was achieved near the electrode edge, and with an increasing disk thickness, the distribution of the electric field near the dielectric surface became more homogenous (Fig. 4). The calculations showed that, when the electrode voltage was
$U= \pm 20 \mathrm{kV}$, an electrostatic field with the amplitudes of the normal components equal to $E_{z 1}= \pm 0.2, E_{z 2}= \pm 1.2$, and $E_{z 3}= \pm 22 \mathrm{kV} / \mathrm{cm}$ for the disks $d_{1}, d_{2}$, and $d_{3}$, respectively, could be produced on the quartz surface. Here, the positive sign of the normal component $E_{z}$ of the dc field corresponds to the negative potential on the electrode. In this case, the electrostatic field drives the electrons back to the surface of the quartz disk.

In the experiments, we measured the incident microwave power and the power reflected from the resonator. Typical waveforms of the incident and reflected powers in the absence of a discharge and with a multipactor are shown in Fig. 5. The tuning of the cavity into the resonance

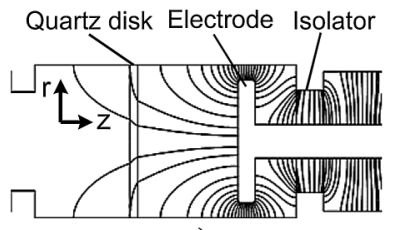

a)

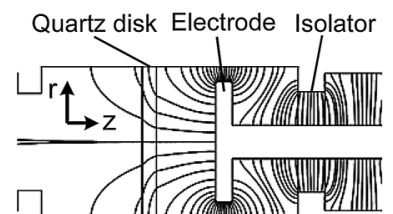

b)

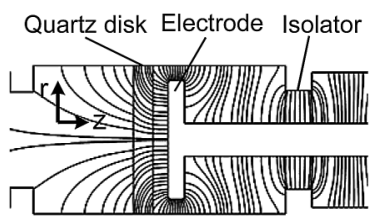

c)

FIG. 4. Distribution of the electrostatic field lines inside the resonator for quartz disks having different thicknesses: (a) $d_{1}=2.3 \mathrm{~mm}$, (b) $d_{2}=4.2 \mathrm{~mm}$, and (c) $d_{3}=6 \mathrm{~mm}$. 


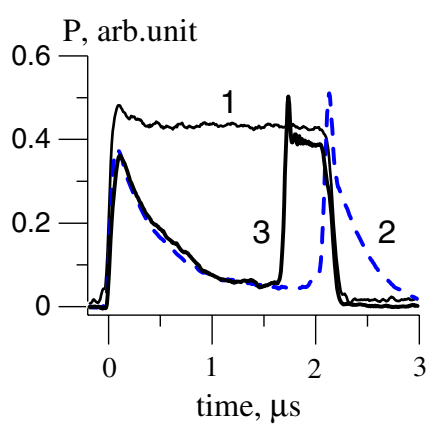

FIG. 5. (Color) Oscillograms of the incident (1) and reflected (2-without multipactor, 3-with multipactor) microwave pulses.

led to a decrease in the reflected microwave power (curve 2 ). The reflected power was minimum when the generator frequency coincided exactly with the eigenfrequency of the cavity. Multipactor occurred when some threshold power was exceeded, and was registered by the changes in the shape of the reflected microwave pulse. Excitation of the discharge led to the detuning of the cavity from the resonance and an abrupt increase in the reflected power (curve $3)$. The calculations showed that the resonant state was lost when the total number of electrons in the multipactor exceeded $N=3-4 \times 10^{9}$. The typical duration of the discharge evolution, which was determined by the duration of the front of the reflected signal, did not exceed $50 \mathrm{~ns}$. The value of the electric field on the dielectric surface at the moment of multipactor initiation (breakdown threshold $\left.E_{\mathrm{br}}\right)$ was determined by measuring the incident and reflected microwave power allowing for the distribution of electric field in the resonator, Fig. 3.

Figure 6 shows the experimental dependence of the threshold intensity $E_{\mathrm{br}}$ of the microwave field on the dielectric surface, at which multipactor was initiated, on the amplitude of the normal component $E_{z}$ of the external electrostatic field for disks with different directions and gradients of the rf potential.

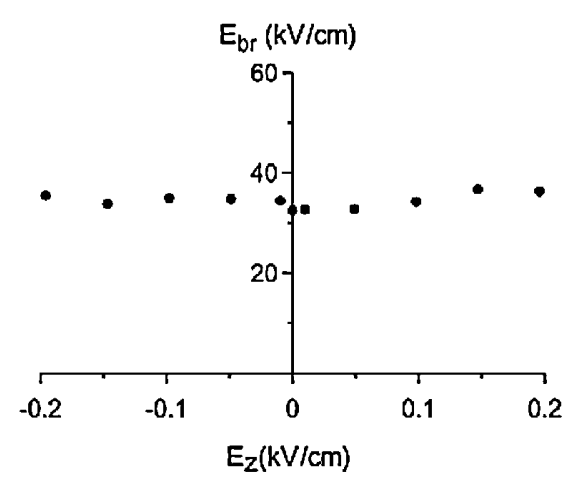

a)

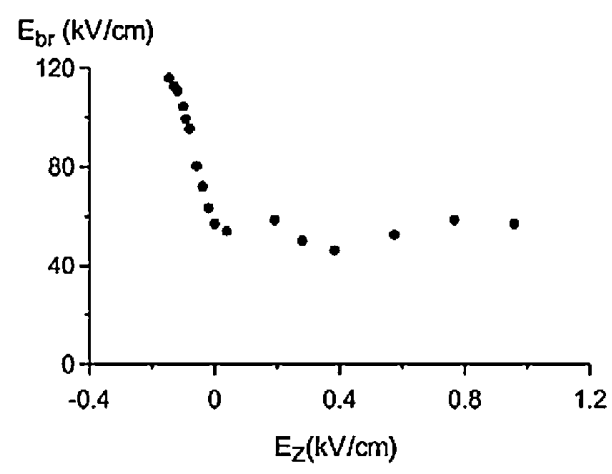

b)
It is seen from the figure that, in the absence of an external dc field $\left(E_{z}=0\right)$, the breakdown threshold increases when one passes over from a disk with the ponderomotive force attracting electrons $\left(F_{\phi}<0\right)$ to a disk with the repulsing force $\left(F_{\phi}>0\right)$. Indeed, in this case, the effective electric field normal to the dielectric surface is determined by only the gradient of the high-frequency potential, $E_{n}=E_{\phi Z}$, and is equal to $E_{\phi Z} \approx-3 ; 0$; or $+10 \mathrm{kV} / \mathrm{cm}$ for the disks $d_{1} d_{2}$, and $d_{3}$, respectively. Note, however, that this field is formed not immediately, but grows gradually from zero up to the maximum, as the microwave energy is stored in the resonator. The typical time of the change in the rf potential gradient is determined by the time of oscillation stabilization in the resonator, $\tau=$ $Q_{L} / \omega \sim 150-200 \mathrm{~ns}$. The effect of the inhomogeneous microwave field on the multipactor was studied in detail in $[16,17]$.

In the experiment, no influence of the external electrostatic field on the multipactor threshold was observed for the disk $d_{1}$, when the ponderomotive force drove the electrons back towards the surface, Fig. 6(a). It might be connected with the fact that the gradient of the rf potential near the surface of this disk reached a value of $-3 \mathrm{kV} / \mathrm{cm}$, whereas the amplitude of the dc field in this experiment was too small and did not exceed $E_{z 1}= \pm 0.2 \mathrm{kV} / \mathrm{cm}$. Therefore, the total external field $E_{n}$ changed insignificantly and did not affect the character of the electron motion near the dielectric surface and, hence, the value of the breakdown threshold.

The experiments showed that in the absence of the ponderomotive force $\left(F_{\phi}=0\right)$, even a weak $\left(E_{z} \sim\right.$ $-200 \mathrm{~V} / \mathrm{cm}$ ) negative dc field resulted in a significant increase in the multipactor threshold, Fig. 6(b). In this case, the breakdown threshold exceeded the maximum amplitude of the electric microwave field $E_{\mathrm{rf}} \sim$ $120 \mathrm{kV} / \mathrm{cm}$, which could be achieved in the experiment with the disk $d_{2}$ and was determined by the power of the microwave generator. Indeed, in this experiment, the elec-

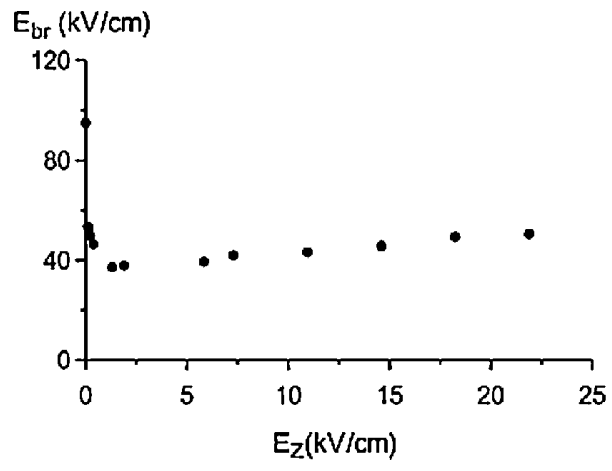

c)

FIG. 6. Dependence of the multipactor threshold on the amplitude of the electrostatic field for quartz disks having different thicknesses and directions of the ponderomotive force, $F_{\phi}$ : (a) $d_{1}=2.3 \mathrm{~mm}, F_{\phi}<0$, (b) $d_{2}=4.2 \mathrm{~mm}, F_{\phi}=0$, (c) $d_{3}=6 \mathrm{~mm}$, $F_{\phi}>0$. 
trostatic force repulsed the electrons away from the dielectric surface, thus preventing the multipactor evolution. It is seen from the Fig. 6(b) that multipactor exists and can be triggered even in the absence of an external static field. In this case the appearance of the electric dc field returning the electrons to the surface is usually associated with accumulation of the charge on the dielectric surface $[14,20]$. Therefore this experiment demonstrates that the positive electric field related to the surface charge is relatively low $(\ll 200 \mathrm{~V} / \mathrm{cm})$ and, hence, does not affect noticeably the results of measuring the breakdown threshold at higher values of the dc fields $E_{\phi Z}$ and $E_{z}$. Note also that under the experimental conditions, when the multipactor occurred steadily in each pulse in the series of microwave pulses with repetition rate $20 \mathrm{~Hz}$, the positive voltage $\left(E_{z}<0\right)$ fed to the electrode resulted at once in the disappearance of the discharge in the next pulse in the series. When the voltage was removed, the multipactor occurred again. Thus, by applying or removing the voltage at the electrode, one could suppress or initiate the multipactor.

At the same time, the positive electric field $\left(E_{z}>0\right)$ changed the threshold of the discharge initiation only insignificantly, excluding the area in the proximity of the point $E_{z}=400 \mathrm{~V} / \mathrm{cm}$, where the threshold decreased by $10 \%-15 \%$, Fig. 6(b). Remember that for $E_{z}>0$, the external dc field drives the electrons back to the surface. In this case, the energy of the returning electrons is determined by both the intensity of the microwave field, and by the time $t^{*}$ of their stay in this field (before they come back to the surface) [14]:

$$
t^{*}=\frac{\sqrt{8 m W_{0}}}{e E_{z}},
$$

where $W_{0} \approx 1-3 \mathrm{eV}$ is the typical energy of the electrons emitted from the dielectric surface. In relatively weak electrostatic fields $\left(0<E_{z}<4 \mathrm{kV} / \mathrm{cm}\right.$, in our case), when the return time $t^{*}$ is long as compared with the $\mathrm{rf}$ field period $\left(\omega t^{*}>1\right)$, the electrons bombard the surface with the energy which is equal to the energy of their natural oscillations in the microwave field near the dielectric surface [14]. In the experiment, it leads to the fact that in the region of the dc field values which ensure that the condition $\omega t^{*}>1$ are fulfilled, the dependence of the breakdown threshold on the value of the electrostatic field becomes weakly pronounced, Fig. 6(b).

In the experiment with the sample $d_{3}$, Fig. 6(c), the ponderomotive force arising in the inhomogeneous rf field repulsed the electrons away from the quartz surface. As a result, the multipactor on this disk did not develop in the absence of the external dc field either. In this case, the breakdown threshold exceeded the maximum field $E_{\mathrm{br}}>$ $90 \mathrm{kV} / \mathrm{cm}$ which could be achieved on the surface of the disk $d_{3}$ and was determined by the power of the microwave generator. Therefore, the negative electrostatic field $\left(E_{z}<\right.$
0 ) did not change the threshold of the multipactor initiation. The change in the polarity of the dc field $\left(E_{z}>0\right)$ resulted in a significant decrease in the breakdown threshold, which decreased to the value equal to $E_{\mathrm{br}}=$ $30 \mathrm{kV} / \mathrm{cm}$ already at $E_{z} \approx 2 \mathrm{kV} / \mathrm{cm}$, Fig. 6(c). Indeed, in this case the electrostatic field counterbalances the potential $\mathrm{rf}$ field $E_{\phi Z}$, so that the resulting field $E_{n}$ becomes positive $\left(E_{n}=E_{\phi Z}+E_{z}>0\right)$ and starts driving the electrons back towards the dielectric surface. As in the previous experiment, here one could suppress or initiate the multipactor by applying or removing the electrode voltage (in this case, having the negative polarity). A further increase in the electrostatic field led to a smooth increase in the breakdown threshold by approximately $15 \%$, which was due, evidently, to a decrease in the energy of the electrons bombarding the dielectric surface, since the duration of their stay in the microwave field $\left(\omega t^{*}<1\right)$ shortened when the electrostatic field increased considerably to be equal to $E_{z}>4 \mathrm{kV} / \mathrm{cm}$.

It should be noted that at present there is no developed theoretical model for the one-side multipactor on dielectric in an inhomogeneous microwave field in the presence of an external dc field, with which the obtained experimental results could be compared quantitatively. For example, in paper [16] the model of a multipactor in an inhomogeneous field of a circularly polarized $\mathrm{TE}_{11}$ mode was developed (in our case, the $\mathrm{TE}_{01}$ mode was used). Additionally, in the model the case of $E_{\mathrm{dc}}=0$ is considered. Therefore we hope that the experimental results presented here will serve as a basis for theoretical activity on this subject.

Thus, the experiments performed showed that one can control the multipactor discharge on a dielectric effectively by suppressing or initiating it. Note that the found effect makes it possible to use such an undesirable phenomenon as a multipactor for practical purposes, e.g., in high-power microwave switches [4] intended to modulate the $Q$ factor in active compressors of microwave pulses.

[1] P. T. Farnsworth, J. Franklin Inst. 218, 411 (1934).

[2] D. H. Preinst, IRE Trans. Electron Devices 8, 243 (1961).

[3] J. G. Power et al., Phys. Rev. Lett. 92, 164801 (2004).

[4] A. L. Vikharev, A. M. Gorbachev, O. A. Ivanov, V. A. Isaev, S. V. Kuzikov, M. A. Lobaev, J. L. Hirshfield, S. H. Gold, and A. K. Kinkead, Phys. Rev. ST Accel. Beams 12, 062003 (2009).

[5] U. Klein and D. Proch, in Proceedings of the Conference on Future Possibilities for Electron Accelerators, Charlottesville, Virginia, 1979, edited by J. S. McCarthy and R. R. Whitney (University of Virginia, Charlottesville, VA, 1979), p. N1.

[6] H. Padamsee et al., IEEE Trans. Magn. 17, 947 (1981).

[7] A. R. Nayaiesh et al., J. Vac. Sci. Technol. 4, 2356 (1986).

[8] S. Michizono et al., J. Vac. Sci. Technol. A 10, 1180 (1992). 
[9] L. L. Hatfield et al., IEEE Trans. Electr. Insul. 24, 985 (1989).

[10] J. Tuckmantel, CERN Technical Report No. 94-26, LEP-2 Notes, Geneva, Switzerland, 1994.

[11] P. Yla-Oijala and M. Ukkola, Nucl. Instrum. Methods Phys. Res., Sect. A 474, 197 (2001).

[12] A. Valfells et al., Phys. Plasmas 7, 750 (2000).

[13] W. D. Moeller et al., Physica C: Superconductivity 441, 229 (2006).

[14] R. A. Kishek and Y. Y. Lau, Phys. Rev. Lett. 80, 193 (1998).

[15] H.C. Kim and J.P. Verboncoeur, Phys. Plasmas 12, 123504 (2005).
[16] M. N. Buynova, V.E. Nechaev, and V.E. Semenov, Radiophys. Quantum Electron. 50, 893 (2007).

[17] M. A. Lobaev, O. A. Ivanov, V. A Isaev, and A. L. Vikharev, Tech. Phys. Lett. 35, 1074 (2009).

[18] A. V. Gaponov and M. A. Miller, Zh. Eksp. Teor. Fiz. 34, 242 (1958).

[19] A. Taflove, Advances in Computational Electrodynamics. The Finite-Difference Time-Domain Method (Artech House, Boston, 1998), p. 724.

[20] R. A. Kishek, Y. Y. Lau, L. K. Ang, A. Valfells, and R. M. Gilgenbach, Phys. Plasmas 5, 2120 (1998). 